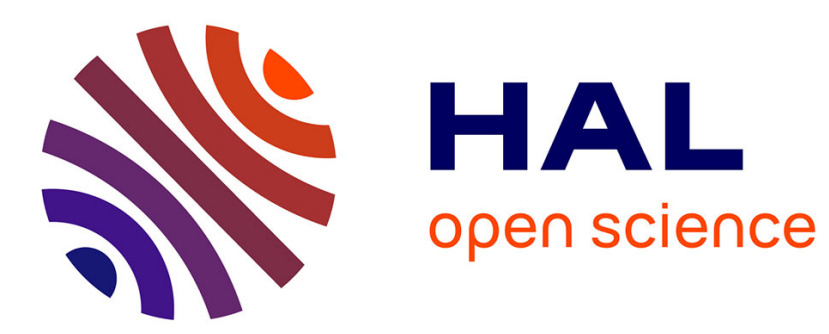

\title{
Discrete symmetries and general relativity, the dark side of gravity \\ Frederic Henry-Couannier
}

\section{To cite this version:}

Frederic Henry-Couannier. Discrete symmetries and general relativity, the dark side of gravity. International Journal of Modern Physics A, 2005, 20, pp.2341-2345. 10.1142/S0217751X05024602 . hal-00003069v8

\section{HAL Id: hal-00003069 \\ https://hal.science/hal-00003069v8}

Submitted on 15 Aug 2005

HAL is a multi-disciplinary open access archive for the deposit and dissemination of scientific research documents, whether they are published or not. The documents may come from teaching and research institutions in France or abroad, or from public or private research centers.
L'archive ouverte pluridisciplinaire $\mathbf{H A L}$, est destinée au dépôt et à la diffusion de documents scientifiques de niveau recherche, publiés ou non, émanant des établissements d'enseignement et de recherche français ou étrangers, des laboratoires publics ou privés. 
International Journal of Modern Physics A

(C) World Scientific Publishing Company

\title{
DISCRETE SYMMETRIES AND GENERAL RELATIVITY, THE DARK SIDE OF GRAVITY
}

\author{
FREDERIC HENRY-COUANNIER \\ CPPM, 163 Avenue De Luminy, Marseille 13009 France. \\ henry@cppm.in2p3.fr \\ Received . \\ Revised ..
}

\begin{abstract}
The parity, time reversal and space/time exchange invariant actions, equations and their conjugate metric solutions are obtained in the context of a general relativistic model modified in order to take into account discrete symmetries. The equations are not covariant and the PPN formalism breaksdown however the new Schwarzschild metric solution in vacuum only starts to differ from that of General Relativity at the Post-Post-Newtonian order in the CMB restframe. Preferred frame gravitomagnetic effects well above expectations of the PPN formalism and within the accuracy of the Gravity Probe B experiment are predicted. No coordinate singularity (black hole) arises in the privileged frame where the energy of gravity is found to vanish. The context is very promising to help the cancelation of vacuum energies as gravitational sources. A flat universe accelerated expansion phase is obtained without resorting to inflation nor a cosmological constant and the BigBang singularity is avoided. The Pioneer anomalous blue-shift is a natural outcome. The context is also promising to help us elucidate several outstanding enigmas such as flat galactic rotation curves or the universe voids. A wave solution is obtained leading to the same binary pulsar decay rate as observed and predicted in GR.
\end{abstract}

Keywords: Negative energy; time reversal, tachyon.

\section{Introduction}

We might summarize the point of view developed in Ref. 2 as follows: any physicist would agree that symmetries are fundamental in contemporary physics. They both allow to constrain the form assumed by the actions and the properties of the basic objects such as fields from which the actions are built and the phenomenology is derived. If we miss some fundamental symmetry of mother nature or misunderstand the way it works, the physical world description we will derive might be either totally wrong or in the best case incomplete. As is well known, a discrete operator such as the time reversal one in Quantum Field Theory may be either unitary or antiunitary. The anti-unitary choice for it is the conventional one, and the commonly accepted derived picture undoubtedly constitutes an important part of our culture. However, our starting point in Ref. 2 was to take serious the other mathematical possibility for the time reversal operator, e.g. that it be unitary and see how far we 
could follow this theoretical option. Then a very different conception of time reversal arises together with negative energy fields. However, at some point we found that there is no way to reach a coherent description in flat space-time. But fortunately, we know that the complete picture should include gravity and this, as we shall show, naturally allows us to achieve our primary goal: understand time reversal and by the way the negative energy representations of the Lorentz group. The reader is referred to Ref. 2 for our investigation of negative energies and time reversal restricted to QFT in flat space-time but an interesting analysis can also be found in Ref. 3 .

\section{Negative Energies in Quantum Field Theory}

Let us gather the main information we learned from our investigation in Ref. 2 of negative energies in Relativistic QFT indicating that the correct theoretical framework for handling them should be a modified GR.

- TheoreticaI Motivations

In second quantization, all relativistic field equations admit genuine negative energy field solutions creating and annihilating negative energy quanta. Unitary time reversal links these fields to the positive energy ones. The unitary choice, usual for all other symmetries in physics, also allows us to avoid the well known paradoxes associated with time reversal. Positive and negative energy fields vacuum divergences we encounter after second quantization are unsurprisingly found to be exactly opposite. The negative energy fields action must be maximised. However, there is no way to reach a coherent theory involving negative energies in flat space-time. Indeed, if positive and negative energy scalar fields are time reversal conjugate, also must be their Hamiltonian densities and actions. In the context of the enriched GR presented here, this is only possible thanks to the new metric transformation under discrete symmetries.

- Phenomenological Motivations

In a mirror negative energy world whose fields remain non coupled to our world positive energy fields, stability is insured and the behaviour of matter and radiation is as usual. Hence, it is just a matter of convention to define each one as a positive or negative energy world. Otherwise, if the two worlds interact gravitationally, promising phenomenology is expected. Indeed, many outstanding enigmas indicate that repelling gravity might play an important role in physics: flat galactic rotation curves, the Pioneer effect, the flatness of the universe, acceleration and its voids, etc... But negative energy states never manifested themselves up to now, suggesting that a barrier is at work preventing the two worlds to interact except through gravity.

- A Modified GR to Circumvent the Main Issues A trivial cancellation between vacuum divergences is not acceptable since the Casimir effect shows evidence for vacuum fluctuations. But the posi- 
tive and negative energy worlds could be maximally gravitationally coupled in such a way as to produce at least exact cancellations of vacuum energies gravitational effects. Also, a generic catastrophic instability issue arises whenever quantum positive and negative energy fields are allowed to interact. If we restrict the stability issue to the modified gravity, this disastrous scenario is avoided. Finally, allowing both positive and negative energy virtual photons to propagate the electromagnetic interaction, simply makes it disappear. The local gravitational interaction is treated very differently in our modified GR so that this unpleasant feature is also avoided.

- Outlooks

A left-handed kinetic and interaction Lagrangian can satisfactorily describe all known physics (except mass terms which anyway remain problematic in modern physics). This strongly supports the idea that the right handed chiral fields might be living in another world (where the 3-volume reversal under parity presumably would make these fields acquire a negative energy density) and may provide as shown in Ref. 2 an interesting explanation for maximal parity violation observed in the weak interaction.

If the connection between the two worlds is fully re-established above a given energy threshold, then loop divergences naturally would get cancelled thanks to the positive and negative energy virtual propagators compensation. Such reconnection might take place through a new transformation process allowing particles to jump from one metric to the conjugate one presumably at places where the conjugate metrics meet each other.

\section{Conjugate Worlds Gravitational Coupling}

From Ref. 4 we learn that if a discrete symmetry (Parity or/and Time reversal) transforms the general coordinates, this will not affect a scalar action however if the inertial coordinates $\xi^{\alpha}$ are also transformed in a non-trivial way:

$$
\xi^{\alpha} \rightarrow \tilde{\xi}^{\alpha},
$$

non-trivial in the sense that in general $\tilde{\xi}^{\alpha} \neq \xi_{\alpha}$, our metric terms will be affected and our action is not expected to be invariant under $\mathrm{P}$ or T. Having two conjugate inertial coordinate systems, we can build, following the usual procedure, two discrete symmetry reversal conjugate metric tensors:

$$
g_{\mu \nu}=\eta_{\alpha \beta} \frac{\partial \xi^{\alpha}}{\partial x^{\mu}} \frac{\partial \xi^{\beta}}{\partial x^{\nu}}, \quad \tilde{g}_{\mu \nu}=\eta_{\alpha \beta} \frac{\partial \tilde{\xi}^{\alpha}}{\partial x^{\mu}} \frac{\partial \tilde{\xi}^{\beta}}{\partial x^{\nu}} .
$$

This means that there exists a coordinate system where the discrete symmetry applies in the trivial way (for instance $x^{0} \rightarrow-x^{0}$ in case of time reversal) transforming the conjugate metrics into one another. We postulate that this coordinate system is such that $\tilde{g}_{\mu \nu}$ identifies with $g^{\mu \nu}$, a non covariant relation making this frame a privileged one. Then, we should distinguish between those fields following the 
geodesics of $\tilde{g}_{\mu \nu}$ and the others following the geodesics of $g_{\mu \nu}$. We first build the total action: sum of usual $I_{R G}$ and the new conjugate one $\tilde{I}_{R G}$. The conjugate actions are separately general coordinate scalars and adding the two pieces is necessary to obtain a discrete symmetry reversal invariant total action. In this system, varying our action, applying the extremum action principle and making use of the relation $\delta g^{\rho \kappa}(x)=-g^{\rho \mu}(x) g^{\nu \kappa}(x) \delta g_{\mu \nu}(x)$ would lead us to a modified Einstein equation (with $c=1$ ) :

$$
\begin{gathered}
-8 \pi G\left(\sqrt{g(x)} T_{\rho \sigma}-\sqrt{g^{-1}(x)} \tilde{T}^{\rho \sigma}\right)= \\
\sqrt{g(x)}\left(R_{\rho \sigma}-\frac{1}{2} g_{\rho \sigma} R\right)-\sqrt{g^{-1}(x)}\left(R^{\rho \sigma}-\frac{1}{2} g^{\rho \sigma} R\right)_{g^{\rho \sigma} \rightarrow g_{\rho \sigma}, g_{\rho \sigma} \rightarrow g^{\rho \sigma}} .
\end{gathered}
$$

This equation, only valid as it stands in our privileged working coordinate system, is not general covariant and not intended to be so. Indeed, it follows from both an extremum principle of our general covariant and discrete symmetry invariant action and a non-covariant relation between one metric and its discrete symmetry reversal conjugate. The straightforward interpretation of the Left hand side is that fields living in the reversed metric world are seen from our world as negative energy density fields but living in the conjugate metric prevents them from interacting with our world fields except through gravitation. However coupling in this traditional way metric fields with matter and radiation fields assumes implicitly that we treat and understand in the same way both directions of the bi-directional talk between matter and gravity. We will see why this is not always possible and in general will only work with the purely gravitational action $I_{G}+\tilde{I}_{G}$. The derived equation is neither unique nor valid in such a general form as above. Indeed, symmetry requirements will a priori determine two possible simplified forms for the conjugate metrics in the privileged coordinate system.

\section{Isotropy and Space/Time Symmetry}

The isotropy condition for both conjugate metrics determines their general form:

$$
d \tau^{2}=B(r, t) d t^{2}+A(r, t) d \sigma^{2}, \quad d \tilde{\tau}^{2}=\frac{1}{B(r, t)} d t^{2}+\frac{1}{A(r, t)} d \sigma^{2} .
$$

We now want to investigate the space/time symmetry in order to understand the tachyonic Lorentz group representation. Indeed, the natural symmetry linking tachyons to bradyons is the transformation reversing the metric signature, hence, following Ref. 121314 transforming space-like coordinates into time-like ones (we call it $\mathrm{x} / \mathrm{t}$ symmetry). This can be achieved by Wick rotating them and leads us to introduce the flipped signature conjugate metric: $\hat{g}_{\mu \nu}(r, t)=-g_{\mu \nu}(r, t)$. More generally, it is natural to allow our metrics to be complex, the phases corresponding to rotations in the complex plane continuously transforming into one another these 
conjugate metrics. The trivial $\mathrm{B}=\mathrm{A}=\mathrm{C}$ Euclidian form manifestly involves time and space coordinates in a symmetrical way, whatever the function $\mathrm{C}(\mathrm{r}, \mathrm{t})$ :

$$
d \tau^{2}=C(r, t)\left(d t^{2}+d \sigma^{2}\right),
$$

But in general we really need the inverse metric to restore the space/time symmetry. Indeed, the most general expression being

$$
\begin{aligned}
d \tau^{2} & =C(r, t)\left(\frac{1}{A(r, t)} d t^{2}+A(r, t) d \sigma^{2}\right), \\
d \tilde{\tau}^{2} & =\frac{1}{C(r, t)}\left(A(r, t) d t^{2}+\frac{1}{A(r, t)} d \sigma^{2}\right) .
\end{aligned}
$$

only the presence of $C(r, t)$ in general breaks down the space / time symmetry so that the symmetry is insured provided the conjugate metrics verify $A(r, t)=$ $B^{-1}(r, t)$. However, in the special case where $\mathrm{A}$ is a pure phase with angle: $\theta(r, t)=$ $\pi / 2$ it is possible to keep the $C(r, t)$ term and restore again the space/time symmetry thanks to the introduction of the opposite conjugate metric $\hat{g}_{\mu \nu}(r, t)=-g_{\mu \nu}(r, t)$. Then we have four conjugate metrics to describe conjugate positive/negative energy worlds as well as tachyonic/bradyonic worlds. Explicitly:

$$
\begin{array}{lc}
g_{\mu \nu}: & d \tau^{2}=C(r, t)\left[d t^{2}-d \sigma^{2}\right] \\
\tilde{g}_{\mu \nu}=g_{\mu \nu}^{-1}: & d \tilde{\tau}^{2}=\frac{1}{C(r, t)}\left[d t^{2}-d \sigma^{2}\right] \\
\hat{g}_{\mu \nu}=-g_{\mu \nu}: & d \hat{\tau}^{2}=C(r, t)\left[d \sigma^{2}-d t^{2}\right] \\
\hat{\hat{g}}_{\mu \nu}=\hat{\tilde{g}}_{\mu \nu}: & d \hat{\tilde{\tau}}^{2}=\frac{1}{C(r, t)}\left[d \sigma^{2}-d t^{2}\right]
\end{array}
$$

Thus apart from the trivial $\mathrm{B}=\mathrm{A}$ form we only have two kinds of metrics: the "Euclidian" ones satisfying $\mathrm{B}=1 / \mathrm{A}$ and the Minkowskian ones with $\mathrm{B}=-\mathrm{A}$. The conjugate of the metric phase is its complex conjugate and the conjugate of the modulus is its inverse. Successive continuous phase rotations of the form $B=1 / \mathrm{A}$ and $\mathrm{B}=-\mathrm{A}$ can transform a real Euclidian into a couple of space/time conjugate real Minkowskian metrics: indeed, with $B=1 / A= \pm i$ phase rotations the Euclidian metric can be transformed into imaginary Minkowskian opposite metrics which under an additional $C(r, t)=1 / i$ phase rotation lead to two real space/time conjugate stationary metrics.

$$
\begin{aligned}
& d \tau^{2}=d \tilde{\tau}^{2}=\left[d t^{2}-d \sigma^{2}\right] \\
& d \hat{\tau}^{2}=d \hat{\tilde{\tau}}^{2}=\left[d \sigma^{2}-d t^{2}\right]
\end{aligned}
$$

\section{The Complex Metrics and their Variations}

In the following sections we will require that either the metric elements are a priori linked by $A(r, t)=-B(r, t)$ or $A(r, t)=B^{-1}(r, t)$ so that a single degree of freedom eventually remains and we investigate the form of its solution in each case. The metric elements being related can no longer be varied independently. Therefore, given any tensor $T_{\mu \nu}$ and for real metric elements making use of $\frac{\delta g_{i^{\prime} i^{\prime}}}{\delta g_{i i}}=\frac{g_{i^{\prime} i^{\prime}}}{g_{i i}}$ and 
$\frac{\delta g_{t t}}{\delta g_{i i}}=\frac{g_{t t}}{g_{i i}}$ in case $\mathrm{B}=-\mathrm{A}$, a typical action variation will be proportional to a scalar trace:

$$
\delta g_{r r} T^{r r}+\delta g_{t t} T^{t t}+\delta g_{\theta \theta} T^{\theta \theta}+\delta g_{\phi \phi} T^{\phi \phi}=\frac{\delta g_{r r}}{g_{r r}} T
$$

while in case $\mathrm{B}=1 / \mathrm{A}, \frac{\delta g_{i^{\prime} i^{\prime}}}{\delta g_{i i}}=\frac{g_{i^{\prime} i^{\prime}}}{g_{i i}}$ and $\frac{\delta g_{t t}}{\delta g_{i i}}=-\frac{g_{t t}}{g_{i i}}$ and the additional minus sign gives us a modified trace:

$$
T_{B=1 / A}=g_{r r} T^{r r}+g_{\theta \theta} T^{\theta \theta}+g_{\phi \phi} T^{\phi \phi}-g_{t t} T^{t t}
$$

We also need the relation between the relative variations of the inverse conjugate metrics needed to obtain the gravitational equation in term of the components of a single metric: $\frac{\delta \tilde{g}_{x x}}{\tilde{g}_{x x}}=-\frac{\delta g_{x x}}{g_{x x}}$ The previous treatment is only valid for a real (pure modulus) metric. But in case the metric and their variations can be complex, we are able to isolate its modulus and phase part equations. Indeed:

- We isolate the metric's modulus equation if we require that the real metric variation satisfies the above relations.

- We isolate the metric's phase equation if we require the complex variations of the conjugate metrics to be complex conjugate. Then, the conjugate metric imaginary variations are opposite in such a way that $\delta \tilde{g}_{x x}=-\delta g_{x x}$ and $\delta g_{i^{\prime} i^{\prime}}=\delta g_{i i}= \pm \delta g_{t t}$ for $\mathrm{B}=-\mathrm{A}$ and $\mathrm{B}=1 / \mathrm{A}$ respectively while the conjugate metrics real variations are equal.

Eventually, we will find ourselves with an equation for the modulus and two for the phase in both $\mathrm{B}=-\mathrm{A}$ and $\mathrm{B}=1 / \mathrm{A}$ cases. The moduli differential equations are obviously unaffected by the terms involving the opposite $\hat{g}_{\mu \nu}$ and $\tilde{\hat{g}}_{\mu \nu}$ metrics since the conjugate opposite metrics have equal moduli. The phase differential equations also keep unaffected provided the actions involving the $\hat{g}_{\mu \nu}$ and $\tilde{\hat{g}}_{\mu \nu}$ phases are defined with negative invariant measures. The moduli describe homogeneous and isotropic backgrounds for both conjugate metrics which necessarily implies their spatial flatness and $\mathrm{r}$ independence. The phases describe the perturbations over this background. The conjugate metric solutions should always transform into one another under $r \rightarrow-r$ and $t \rightarrow-t$.

\section{6. $B=1 / A$, The Phase: The Schwarzschild Solution}

With $\mathrm{B}=1 / \mathrm{A}$ pure phase metric elements, defining $\mathrm{g}$ to be the modulus of the metric determinant, its square root simplifies and we obtain the following equations in vacuum:

$$
A\left(g_{r r} R^{r r}+g_{\theta \theta} R^{\theta \theta}+g_{\phi \phi} R^{\phi \phi}\right)-B g_{t t} R^{t t} \pm i n v=0
$$

where inv denotes the same expression with the metric elements being everywhere replaced by their inverse and the plus or minus signs refer to the case where the 
variation is respectively real or imaginary. This yields:

$$
\begin{gathered}
4 \frac{A^{\prime \prime}}{A}-4\left(\frac{A^{\prime}}{A}\right)^{2}+8 \frac{A^{\prime}}{A r}=0 \\
\dot{A}=0
\end{gathered}
$$

We easily get the unique static phase solution

$$
1 / B=A=e^{\frac{2 i M G}{r}}
$$

This Euclidian metric is not appropriate to get the geodesics followed by test masses. Rather should we first perform a Wick rotation of the spatial coordinates $r \Rightarrow i r$ to obtain the real Minkowskian metric with components B, -A satisfying:

$$
\begin{gathered}
\Rightarrow-A=e^{\frac{2 M G}{r}} \approx 1+2 \frac{M G}{r}+2 \frac{M^{2} G^{2}}{r^{2}}, \\
\Rightarrow B=-\frac{1}{A}=e^{-\frac{2 M G}{r}} \approx 1-2 \frac{M G}{r}+2 \frac{M^{2} G^{2}}{r^{2}}-\frac{4}{3} \frac{M^{3} G^{3}}{r^{3}} .
\end{gathered}
$$

different from the GR one though in good agreement up to Post-Newtonian order. No black hole type singularity arises in our isotropic system. The conjugate metrics can be transformed into one another through $r \rightarrow-r$ or $M \rightarrow-M$. We could show in Ref. 2 that a left-handed Lagrangian could satisfactorily describe all known physics (at least kinetic and interaction terms) and provide an interesting explanation for maximal parity violation. This strongly supports the idea that the right handed chiral fields are living in another metric and acquire thanks to the 3 -volume reversal a negative energy density.

- Stability

The phenomenology is simple: objects living in the same metric attract each other. Objects living in different metrics repel each other, as if the object living in the conjugate metric contributes as a negative energy source from the point of view of our metric. Eventually, because the solution is instantaneous in the privileged coordinate system (was Newton right?) the usual stability issues reviewed in Refs. 5-8 are avoided. The instability is usually seen in the phenomenology of a positive energy mass interacting with a negative energy mass through an interaction propagated by positive energy virtual interaction particles. The negative energy object is being attracted by the positive energy object, the latter being repulsed by the former. They then accelerate together for ever this resulting in an obviously instable picture. But here the gravitational interaction of two masses living in different metrics exhibits no such instability since they just repel each other. Yet, from the point of view of each metric, this is really the interaction between a positive energy mass and a negative energy mass.

- The Metric for an Extended Source 
To obtain the metric generated by an extended source we postulate that there exists a unique privileged frame where it is possible to combine multiplicatively the metric elements corresponding to all isotropy centers in the $\mathrm{B}=1 / \mathrm{A}$ form. Thus an unusual and amazing feature of the model is that even for moving sources relative to the unique privileged coordinate system (presumably the CMB rest frame), the metric generated is in the static and isotropic form as if we were taking a photo and considering the static picture we get at this time to be the genuine source distribution. Of course, it is not possible to span $3 \mathrm{~d}$-space with isotropic elementary volumes so that having determined the energy momentum tensor of a source distribution inside a given (necessarily non isotropic) 3d-space cell and computed its trace, we must recover isotropy by postulating that this source cell trace contribution is concentrated in a point. Therefore, we probably have two modes of space-time: the continuous Minkowskian one where we have to define an energy momentum tensor for our sources and understand how fields move under the influence of gravity, and a discrete one where instantaneous gravity in Euclidian space-time takes place allowing to derive the Schwarzschild potential out of each individual cell. Eventually, we can divide any source distribution into cells having a mass point source (isotropic and static in the privileged frame) behaviour where the above Schwarzschild treatment always applies in vacuum.

- SEP and Gravitational Energy

The weak equivalence principle is obviously not menaced since once the metric field solution is established, matter and radiation will have to follow its geodesics as in GR. But, because of the non covariance of our equation, a violation of the strong equivalence principle arises at the PPN level for a point-mass source. We are able to compute gravitational energy following the standard method using the pseudo-energy momentum tensor. It vanishes thanks to the relation $\mathrm{B}=1 / \mathrm{A}$ everywhere out of the discret network of sources in the privileged coordinate system. Thus we dont need to show that it should fall in the same way as any other form of energy in a gravitational field and the strong equivalence principle is obviously not menaced. Notice that the pseudo energy-momentum tensor does not happen to be meaningful out of the privileged coordinate system. Indeed, in our framework this object is not a Lorentz tensor.

- Gravitomagnetism

Let us now explore the gravitomagnetic sector of the model. In a chosen PPN coordinate system moving at velocity $w_{i}$ relative to the privileged one, the Lorentz transformed $g_{0 i}$ metric element to Post-Newtonian order for a point mass source $m$ is:

$$
g_{0 i} \approx-4 w_{i} \frac{m}{r}
$$


while the expression in GR of the same metric element involves the sources velocities and angular momenta in the PPN system (see Clifford M.Will: theory and experiment in gravitational physics, p104). As a result, the precession of a gyroscope's spin axis $\mathbf{S}$ relative to distant stars as the gyroscope orbits the earth given by ( C M.Will, p208):

$$
\frac{d S}{d \tau}=\Omega \times S
$$

where

$$
\Omega=\Omega_{\text {geodetic }}+\Omega_{\mathrm{PF}}
$$

involves in addition to the geodetic precession also expected in GR, a preferred frame effect in $\Omega_{\mathrm{PF}}=-\frac{1}{2} \nabla \times g=-2 w \times \nabla\left(\frac{m}{r}\right)$. Hence, not only do we have a new kind of Post Newtonian effect which could not have been accounted for in the Post Newtonian formalism, but this comes in place of the Lense-Thirring precession or "the dragging of inertial frames" interpreted as a genuine coupling in GR between the spins of the earth and gyroscope. It is instructive to compare our effect to the preferred frame effect that arises in the Parametrized Post Newtonian formalism:

$$
\Omega_{\mathrm{PF}}=-\frac{1}{4} \alpha_{1} w \times \nabla\left(\frac{m}{r}\right)
$$

Following C M.Will, p209, for an earth orbiting satellite, the dominant effect comes from the solar term leading to a periodic angular precession with a one year period, with amplitude:

$$
\delta \theta_{\mathrm{PF}} \leq 5 \cdot 10^{-3 \prime \prime} \alpha_{1}
$$

thus completely negligible according to the PPN formalism given the experimental limit $\alpha_{1}<4.10^{-4}$, while in our case:

$$
\delta \theta_{\mathrm{PF}} \leq 0.04^{\prime \prime}
$$

well reachable with the experimental accuracy $\left(5 \cdot 10^{-4 \prime \prime} /\right.$ year $)$ of the Gravity Probe B experiment designed to measure for the first time gravitomagnetism isolated from other Post-Newtonian effects.

Most of the previous analysis relies on the postulated existence of a single privileged coordinate system. It might be, however, that the correct way to understand our instantaneous interaction is to postulate the existence of many comoving coordinate systems. Following this approach is more difficult because combining various metrics after Lorentz transformation into a single object that would also behave as a metric is not possible in general. Moreover, having an instantaneous interaction from the point of view of the metric sources only is expected to generate severe causality violations. 


\section{7. $\mathrm{B}=-\mathrm{A}=\mathrm{h}$ : The Phase: Gravitational Waves}

The metric a priori takes the form:

$$
\begin{aligned}
d \tau^{2} & =h(r, t)\left[d \sigma^{2}-d t^{2}\right] \\
d \tilde{\tau}^{2} & =h^{*}(r, t)\left[d \sigma^{2}-d t^{2}\right]
\end{aligned}
$$

And our modified Einstein equation yields in vacuum:

$$
A\left(g_{r r} R^{r r}+g_{\theta \theta} R^{\theta \theta}+g_{\phi \phi} R^{\phi \phi}-g_{t t} R^{t t}\right) \pm i n v=0
$$

or

$$
\begin{gathered}
\square^{2} h-h^{2}+\dot{h}^{2}=0 \\
-h^{\prime 2}+\dot{h}^{2}=0
\end{gathered}
$$

Only plane wave pure phase conjugate solutions transforming as required into one another through time and space reversal are acceptable. In general a superposition of such plane wave solutions is not a phase and is not solution of the quadratic equation. For instance the spherical wave cannot be accepted as a solution for our equation but if the elementary wave solutions are one-dimensional topological defects with arbitrary directions, each isotropic cluster of such superposition (which do not spatially overlap) of one dimensional plane wave solutions produced with a common frequency by a quantum of generic impulse source term $n \pi G \delta T=\frac{\delta(r) \delta(t)}{A}$ can be approximated by a continuous spherical wave on large scales so that the computation of the lost energy will proceed as in GR. This sector of cosmic waves being completely linear, the energy carried by a wave is not a source for other waves. The superposition of an outgoing and ingoing waves with the same frequencies is a standing wave. Because it is not a phase, it can contribute in the moduli equation only and turns out to be a source term. On large scales the averaged $\dot{h}^{2}-h^{\prime 2}$ should vanish while on small space-time scales relative to the wavelength, the Zitterbewegund of $\dot{h}^{2}-h^{\prime 2}$ is the perturbation needed to start a non stationary evolution, i.e. for the birth of a couple of time reversal conjugate universes which scale factors are the evolving moduli as we shall show in the next section.

We shall now predict the same energy lost through gravitational waves radiation of the binary pulsar as in GR in good agreement with the observed decay of the orbit period. We follow Weinberg's computation of the power emitted per unit solid angle and adopt the same notations. For any extended non relativistic source the solution of $\square^{2} h(r, t)=n \pi \frac{G}{6} \delta(r) \delta(t)$ is the retarded potential:

$$
h(x, t)=n \pi \frac{-G}{4 \pi} \frac{1}{6} \int d^{3} x^{\prime} \frac{\delta T_{00}\left(x^{\prime}, t-\left|x-x^{\prime}\right|\right)}{\left|x-x^{\prime}\right|}
$$

The radial momentum component of our gravitational wave energy momentum tensor reads:

$$
T_{r 0}=\frac{1}{n \pi G} \sum_{\sigma=0,3} \frac{\partial R}{\partial\left(\frac{\partial A_{\sigma}}{\partial r}\right)} \frac{\partial A_{\sigma}}{\partial t}=\frac{1}{n \pi G} \frac{\partial R}{\partial\left(\frac{\partial h}{\partial r}\right)} \frac{\partial h}{\partial t}
$$


We have used here an energy-momentum pseudo-tensor different from the usual one used in GR. It exploits the Lorentz invariance of both our action and the relation between conjugate metrics in the privilege coordinate system. Indeed, both the $\mathrm{B}=-\mathrm{A}$ metric and its inverse are Lorentz invariant starting from the privileged coordinate system.

Replacing by the expression of our wave solution,

$$
\begin{aligned}
& \forall \sigma, A_{\sigma}=h=\sum_{\omega, k} h(\omega, k) e^{i(\omega t-k r)}+h^{*}(\omega, k) e^{-i(\omega t-k r)} \\
& \left\langle T_{r 0}(\omega, k)\right\rangle=\frac{6}{n \pi G}(-2)\left\langle h^{\prime} \dot{h}\right\rangle_{\omega, k}=\frac{6}{n \pi G} 4 \omega^{2}|h(\omega, k)|^{2}
\end{aligned}
$$

We find that the power emitted per unit solid angle in the direction $k$ is:

$$
\begin{aligned}
& \frac{d P}{d \Omega}(\omega, k)=r^{2}\left\langle T_{r 0}(\omega, k)\right\rangle=r^{2} \frac{24}{n \pi G} \omega^{2}|h(\omega, k)|^{2} \\
& \quad \frac{d P}{d \Omega}(\omega, k)=24 \frac{\omega^{2}}{n \pi G}\left(n \pi \frac{-G}{4 \pi} \frac{1}{6}\right)^{2} \delta T_{00}^{2}(\omega, k) \\
& \quad=\frac{n}{24} \frac{\omega^{2} G}{\pi} \delta T_{00}^{2}(\omega, k) \\
& \quad=\frac{2}{\pi} G \omega^{2} \delta T_{00}^{2}(\omega, k) \\
& \quad=\frac{G \omega^{6}}{2 \pi} k_{i} k_{j} k_{l} k_{m} D_{i j}(\omega) D_{l m}(\omega)
\end{aligned}
$$

Where $\mathrm{n}$ has been given the numerical value 48 taking account of the fact that in the Newtonian limit our equation $6 \square^{2} h=48 \pi G \delta T$ must give again $\nabla^{2} g_{00}=$ $-8 \pi G T_{00}$ (the same straightforward reasoning allows to determine $\mathrm{n}$ in all the other gravitational equations of the theory!). Then following Weinberg, we may write in terms of the moment of inertia Fourier transforms in the observer coordinate system:

$$
\begin{aligned}
& P=\frac{2}{15} G \omega^{6}\left[\left(D_{i i}(\omega)\right)^{2}+2 D_{i j}(\omega) D_{i j}(\omega)\right] \\
& P=\frac{2}{15} G \omega^{6} 12 D_{11}^{2}(\omega)
\end{aligned}
$$

For a rotating body with angular speed $\Omega$, equatorial ellipticity e, moment of inertia I in the rotating coordinates, $\omega=2 \Omega, D_{11}(\omega)=\frac{e I}{4}$ and the radiated power reads:

$$
P=2 \frac{12}{15} G 64 \Omega^{6} e^{2} \frac{I^{2}}{16}=\frac{32}{5} G \Omega^{6} e^{2} I^{2}
$$

as in General Relativity. The main difference is that our gravitational wave is found to propagate pure monopole modes linked by $-g_{00}=g_{11}=g_{22}=g_{33}=h$. But these cannot be excited independently whatever the source configuration since we have the single degree of freedom $\mathrm{h}(\mathrm{r}, \mathrm{t})$. Quantifying the $\mathrm{h}$ field must generate a new gravitational propagated interaction in addition to the Schwarzschild non propagated solution we obtained in the previous section. We cannot add its potential to the exponential Schwarzschild one since this would severely conflict with observations. Thus the two solutions do not cohabit. Rather the Schwarzschild solution is valid up to a critical distance (probably related to the value of the quantum of gravitational energy carried by the waves) where the other one takes over. 


\section{8. $\mathrm{B}=-\mathrm{A}$ : The Modulus: Cosmology}

The gravity for moduli, i.e. global gravity (this is cosmology) only depends on time. Indeed, in a global privileged coordinate system, a couple of time reversal conjugate purely time dependent solutions can be derived from a new couple of conjugate actions. The existence of a time reversal conjugate universe was also suggested a long time ago in Ref. 9. Notice that even if we did not impose the $\mathrm{B}=-\mathrm{A}$ condition, the only possible privileged coordinate system with both metrics spatially homogeneous and isotropic would be a flat Cartesian one. When $\mathrm{B}=-\mathrm{A}$, our equation for gravity now reads:

$$
\sqrt{A^{4}} R-\frac{1}{\sqrt{A^{4}}} \tilde{R}=0
$$

with $\tilde{R}=R_{A \rightarrow 1 / A}$. For a cosmological homogeneous source we will get purely time dependent background solutions and can keep only the time derivatives leading to:

$$
3 A\left(-\frac{\ddot{A}}{A}+\frac{1}{2}\left(\frac{\dot{A}}{A}\right)^{2}\right)-\frac{3}{A}\left(\frac{\ddot{A}}{A}-\frac{3}{2}\left(\frac{\dot{A}}{A}\right)^{2}\right)=0
$$

The solutions for such always null right hand side describe worlds where only light can live (null source trace). The trivial $\mathrm{A}=1$ stationary solution thus describes a self conjugate light world. A mass perturbation $\delta(t)$ (as a superposition of ingoing and outgoing GWs) is needed for the birth of times to take place and see a couple of universes start evolving. The background matter worlds have simple evolution laws in the particular ranges $A<<1, A \approx 1, A>>1$. Indeed, the scale factor evolution is then driven (here non dimensional time unit is used) by the following differential equations in the three particular domains:

$$
\begin{gathered}
a<<1 \Rightarrow \ddot{a} \propto \frac{3}{2} \frac{\dot{a}^{2}}{a} \Rightarrow a \propto 1 / t^{2} \text { where } t<0, \\
a \approx 1 \Rightarrow \ddot{a} \propto \frac{\dot{a}^{2}}{a} \Rightarrow a \propto e^{t}, \\
a>>1 \Rightarrow \ddot{a} \propto \frac{1}{2} \frac{\dot{a}^{2}}{a} \Rightarrow a \propto t^{2} \text { where } t>0 .
\end{gathered}
$$

We can check that $t \rightarrow-t$ implies $1 / t^{2} \rightarrow t^{2}$ but also $e^{t} \rightarrow e^{-t}$ thus $A \rightarrow 1 / A, B \rightarrow$ $1 / B$ when $t$ reverses as required. Let us stress that the couple of cosmological metric solutions does not imply any local gravitational interaction between objects but only a global one between the two conjugate universes as in Ref. 10. A striking and very uncommon feature is that the evolution of the scale factor is mostly driven by the gravitational energy exchange between the coupled universes independently of the universes matter and radiation content. In particular, the observed flatness can no longer be translated into the usual estimation $\Omega_{m}=1$ from the WMAP data. The $t^{2}$ evolution is one of the very few possibilities. Thus, we are most probably living in a constantly accelerating universe. Our and the conjugate universe crossed each 
other and two reversed time parameters appeared at their birth time. At last, not only our universe is accelerated without any need for a cosmological constant or dark energy component but it is flat without inflation and gets rid of the big-bang singularity. The vanishing of cosmological constant terms provided

$$
d^{4} \xi(x) \Lambda=d^{4} \tilde{\xi}(x) \tilde{\Lambda} .
$$

appears to be only a local issue (for our Schwarzschild solution).

\section{9. $\mathrm{B}=1 / \mathrm{A}$ : The Modulus: Pioneer Effect and Pseudo-Horizon}

\subsection{The Pioneer Effect}

When $\mathrm{B}=1 / \mathrm{A}$, our equation for the background takes a very simple form:

$$
\frac{3}{2}\left(B-\frac{1}{B}\right)\left(\frac{\dot{B}}{B}\right)^{2}=0
$$

implying $1=\mathrm{B}=1 / \mathrm{A}$. However, the unit element here remains to be defined. We require the unit element to be such that an object at rest $(d \sigma=0)$ will not feel any gravitational discontinuity when passing from the $\mathrm{B}=-1 / \mathrm{A}$ regime to the $\mathrm{B}=-\mathrm{A}$ regime. Thus the $\mathrm{B}$ element must be the same for both $\mathrm{B}=-\mathrm{A}$ and $\mathrm{B}=-1 / \mathrm{A}$ metrics. At a particular distance from a massive body, the cosmological metric flips from a $\mathrm{A}=-\mathrm{B}$ solution to a $\mathrm{A}=-1 / \mathrm{B}$ solution. This results in the photons being red shifted compared to wavelengths emitted by atomic references in the $\mathrm{A}=-\mathrm{B}$ expanded regime (it is more accurate to say that the reference periods contract while the photon keeps unaffected) while they will be blue shifted with exactly the same magnitude as compared to the same references in the $A=-1 / B$ regime (it is more accurate to say that the reference periods still contract while the photon periods contract twice more) . Then, perhaps we should not be surprised to receive the

photons from an object at several Astronomical Units as are the Pioneer aircrafts slightly blue-shifted, an effect which according to Ref. 11 has been measured to a very good precision with the expected magnitude. The space-space metric component discontinuity when passing from the $\mathrm{B}=-\mathrm{A}$ to the $\mathrm{B}=-1 / \mathrm{A}$ regime might transmit and deviate part of the photons and reflect the others as a genuine gravitational mirror. Alternatively, we could have required that the space-space element is the continuous one from one regime to the other and also got (this is easy to check) the correct red shifts and blue shifts provided $\mathrm{A}=-\mathrm{B}$ are in contraction. But in this case, we get discontinuities of the metric for both matter and light. A discontinuity of the gravitational field is expected to produce caustics where matter is unexpectedly concentrated along rings.

\subsection{The Black Hole Horizon}

Our exponential solution tells us that there is no more BH singularity in our theory. However a test mass approaching the Schwarzschild radius of a massive body at rest 
with respect to the global coordinate system is propagating in a modulus and phase originating superposition:

$$
d \tau^{2}=\frac{1}{A(r) a(t)^{2}} d t^{2}-A(r) a(t)^{2} d \sigma^{2} .
$$

But for a strong enough gravitational field, e.g probably not much below the Schwarzschild radius thanks to the exponential regime, the $\mathrm{A}(\mathrm{r})$ term is expected to "cross" the scale factor term so that locally

$$
d \tau^{2}=d t^{2}-d \sigma^{2} .
$$

and a better space/time symmetry (hence a more stable configuration) is achieved. Reaching this radius a local spontaneous phase rotation of the metric can transfer the test mass in the Euclidian world and possibly via an extra rotation in the space/time conjugate light world. As well as a genuine black hole horizon this mechanism would account for the absence of thermonuclear bursts from $\mathrm{BH}$ candidates.

\section{Test mass motion}

The model developed up to know only modified the way matter sources affect geometry. Remains to be clarified how various fields should move under the influence of various metrics. In the $\mathrm{B}=-\mathrm{A}$ regime, we can adopt the usual action minimally coupling radiation and matter fields to the non dynamical metric as obtained from an additive superposition of our gravitational equations solutions. In the $B=1 / A$ regime, postulating a multiplicative superposition of local metrics solutions before applying the Wick rotation is only possible if there exists a single privileged coordinate system. Indeed, in general the multiplicative superposition of metrics will only generate a metric provided we have a common privileged system. If this is not the case, perhaps should we give up the hope to combine the metrics and minimally couple the resulting metric with matter and radiation in the usual way.

\section{Phenomenological Outlooks}

We now show that this dark gravity model provides a very powerful alternative to dark matter models.

\subsection{Structure Formation and the Early Universe}

Following an original idea first proposed by JP Petit in Ref 1, it is very tempting to interpret the universe voids as being filled with invisible matter living in the conjugate metric and repelling our matter at the frontier of what eventually appears to us as empty bubbles. On the other hand, a massive structure such as a galaxy in our metric repels the matter living in the conjugate metric creating there an attracting void for the galaxy which might help to explain the flat rotation curves and gravitational lensing effects. 
Taking the dominating mass density contribution to be the baryonic matter well established density $\left(\rho_{0}=2.10^{-31} \mathrm{~g} / \mathrm{cm}^{3}\right)$ let us just assume that the crossing between the conjugate universes took place nearly at the time $t_{R}$ of hydrogen recombination where the densities equilibrium could be momentarily realised between the conjugate universes. On our side we obtain the density at this early time by:

$$
\begin{aligned}
& \rho\left(t_{R}\right)=\rho\left(t_{0}\right)\left(\frac{a\left(t_{0}\right)}{a\left(t_{R}\right)}\right)^{3} \\
& =\rho\left(t_{0}\right)\left(1+z_{R}\right)^{3}=\rho\left(t_{0}\right)(1500)^{3}
\end{aligned}
$$

which were also the density in the conjugate universe. Also, we link the Hubble parameter at $t_{R}=0$, its value at the transition time $t_{T}$ between the constantly accelerated phase and the previous exponential phase and nowadays value at $t_{0}$ through:

$$
\frac{H\left(t_{0}\right)}{H\left(t_{R}\right)} \approx \frac{H\left(t_{0}\right)}{H\left(t_{T}\right)}=\left(\frac{a\left(t_{T}\right)}{a\left(t_{0}\right)}\right)^{1 / 2}
$$

thus:

$$
H\left(t_{R}\right) \approx H\left(t_{0}\right)(1500)^{1 / 2}
$$

opposite to the corresponding Hubble parameter in the conjugate side. Neglecting the effect of the universe expansion in the evolution equation of density fluctuations $\delta(t)$ on our side and making use of $p \ll \rho$ after recombination leads to the following differential equation:

$$
\ddot{\delta}-4 \pi G \rho \delta=0
$$

and the exponentially growing fluctuations:

$$
\delta_{+}=e^{\sqrt{4 \pi G \rho} t}
$$

to be compared with the universe exponential expansion

$$
a(t)=e^{H_{R} t}
$$

We can check that:

$$
\frac{H_{R}}{\sqrt{4 \pi G \rho_{R}}}=\sqrt{\frac{2}{3}} \frac{1}{1500} \sqrt{\frac{\rho_{c}}{\rho_{0}}} \approx 4.10^{-3}
$$

insuring that the universe expanding rate is indeed negligible compared to the density fluctuations growing rate thereby justifying our previous approximation. At the end of the exponential phase,

$$
t_{T} \approx 1 / H_{R} \Rightarrow \frac{\delta_{+}\left(t_{T}\right)}{\delta_{+}(0)}>>10^{5}
$$

so that we could for sure reach the non-linear regime during this early universe epoch even starting from the $10^{-5}$ density fluctuations of the CMB. By the way, notice that the absence of gravitational horizon up to sufficiently large distances might well account for the large scale homogeneity of the CMB without any need 
for inflation. The typical mass of a fluctuation after recombination if the present universe density is $\rho_{0}=2.10^{-31} \mathrm{~g} / \mathrm{cm}^{3}$ is the Jean mass $\approx 10^{8} M_{\odot}$ approaching fairly well the typical galaxy baryonic visible mass of $\approx 10^{9} M_{\odot}$. In the conjugate universe we started the contracting regime at a temperature a little bit greater than the recombination temperature so that the Jean mass were as large as $\approx 10^{19} M_{\odot}$. Because the conjugate universes had comparable densities near crossing time, the ratio between the primordial inhomogeneities radii in the conjugate universes is roughly $10^{11 / 3}$. Fortunately, this also approaches the ratio between the radius of a typical void (conjugate universe over-density) and radius of a galaxy (our universe structure) as expected since the subsequent expansion of the universe should not affect these ratios.

Therefore, we found that our model is not only successful in explaining the growing of the very small initial CMB fluctuations in the linear regime without any need for dark matter nor dark energy but also leads to the correct typical sizes of both galaxies and the universe voids interpreted as over-densities in the conjugate metric (in a radiative regime). In this derivation the non singular behaviour of the metric and its very slow expanding rate at the beginning of time played a crucial role.

\subsection{Flat Galaxy Rotation Curves?}

The subsequent non-linear evolution is also facilitated given that a twice older universe $(2 / \mathrm{H} 0=28$ billion years) to be compared with the oldest galaxies ages $(\mathrm{z}=5)$ $\approx 17$ billion years in quite a good agreement with the oldest stars ages provides more time for galaxy formation. The interactions between conjugate density fluctuations might also solve the galaxy missing mass problem. Indeed, the presence of the galactic structure should generate a large and smoothly varying void in the negative energy universe (where we are in a radiative regime) having exactly the same effect as a massive positive energy huge halo. This eventually could only works thanks to the gravitational superposition principle of this dark gravity model. Here, there is no equivalent of the Birkhov theorem. It remains to be checked that the shape of such halo is suitable to obtain our flat galactic rotation curves and the correct star velocities in the vicinity of the center of the galaxy. If not, the concentration of negative energy matter in a shell that surrounds the galaxy (at the metric discontinuity) might also help its rotation (see Ref 1). At last, if we take into account the effective large mass induced by the Dark Side, our galaxies are much heavier and extend very far away from the visible part (which in some cases might be too faint to be detected), so that the missing mass seen at the level of clusters of galaxies could also be explained. Eventually our hope to be as successful as the standard model in cosmology while avoiding ad-hoc hypothesis and adjustable parameters such as a cosmological constant, cold dark matter, inflation seems on a very good way so far. 


\section{Conclusion}

We could settle down here the foundations for a modified theory of gravitation. This theory is essentially general relativity enriched to take into account the fundamental discrete symmetries involved in the structure of the Lorentz group. Eventually, we find that this allows to solve many long lasting theoretical issues such as negative energies and stability, QFT vacuum divergences and the cosmological constant but also leads to very remarkable phenomenological predictions: Locally, the disagreement with GR only arises at the PPN level in the cosmological rest frame, black holes disappear and gravitomagnetism arises in an unusual way. Globally (this is cosmology), a constantly accelerating necessarily flat universe in good agreement with the present data is a natural outcome of the model. The formation of structures in this dark gravity theory works well without any need for dark energy nor dark matter component, and the context is very promissing to help solving the galaxy or cluster of galaxies missing mass issues. At last, we could also show that the space/time exchange symmetry clarifies the status of tachyonic representations and allows us to derive a gravitational wave solution leading to the observed decay of the binary pulsar orbital period. 


\section{References}

1. JP. Petit,

2. F. Henry-Couannier, gr-qc/0404110.

3. S. Chen, hep-th/0203230.

4. S. Weinberg, Gravitation and Cosmology (John Wiley and sons, New York, 1972).

5. A. D. Linde, Rept. Prog. Phys. 47, 925 (1984).

6. R. R. Caldwell, Phys. Rev. Lett. 91, 071301 (2003).

7. P. H. Frampton, Mod. Phys. Lett. A19, 801 (2004).

8. S. M. Carrol, M. Hoffman and M.Trodden Phys. Rev. D68, 023509 (2003).

9. A. D. Sakharov, JETP Lett. 5, 24 (1967).

10. A. D. Linde, Phys. Lett. B200, 272 (1988).

11. J. D. Anderson, Phys. Rev. D65, 082004 (2002).

12. M. Tegmark, gr-qc/9702052

13. G. Feinberg, Phys. Rev. D17, No 6.

14. E. Recami, arXiv:physics/0101108. 